\title{
Cranial reduction and fixation with a resorbable plate combined with cerebrospinal fluid shunting for difficult-to-manage macrocephaly related to hydrocephalus
}

\author{
Case report
}

\author{
Jotham C. Manwaring, M.D., ${ }^{1}$ Devon Truong, P.A., ${ }^{2}$ Armen R. Deukmedjian, M.D., ${ }^{1}$ \\ Carolyn M. Carey, M.D., M.B.A., ${ }^{2}$ Bruce B. Storrs, M.D., ${ }^{2}$ Luis F. Rodriguez, M.D., ${ }^{2}$ \\ Lisa Tetreault, R.N., C.C.R.P., ${ }^{2}$ ANd Gerald F. Tuite, M.D. ${ }^{2}$ \\ ${ }^{1}$ Department of Neurosurgery and Brain Repair, University of South Florida Morsani College of Medicine, \\ Tampa; and ${ }^{2}$ Neuroscience Institute, All Children's Hospital/Johns Hopkins Medicine, St. Petersburg, Florida
}

\begin{abstract}
The management of newborns with extreme macrocephaly related to hydrocephalus can be difficult; balancing the treatment of severe cranial deformity with optimal hydrocephalus management can be complicated. Excessive CSF drainage can result in significant suture overlap that leads to difficulties in patient positioning, secondary synostosis, and long-term aesthetic complications. Delayed cranial reduction and remodeling procedures carry significant risk, and the aesthetic outcomes have sometimes been poor.

The authors describe a newborn with severe macrocephaly who underwent shunt placement followed by a limited cranial reduction and fixation procedure using an absorbable plate within the 1st week of life. The procedure produced an immediate intracranial volume reduction of $49 \%$. This novel management strategy facilitated patient positioning, simplified hydrocephalus management, and provided an excellent aesthetic outcome. (http://thejns.org/doi/abs/10.3171/2012.10.PEDS12340)
\end{abstract}

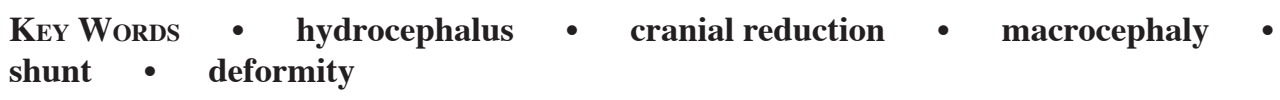

\begin{abstract}
$\mathrm{S}$ EVERE cranial deformity can occur after shunt placement in newborns with extreme hydrocephalus. Deformity frequently develops due to CSF overdrainage. This problem can be lessened with the use of programmable shunts, high-pressure valves, or flowcontrol devices, with or without the use of a cranial orthotic helmet and vigilant repositioning. ${ }^{1}$ However, despite these efforts, hydrocephalic newborns with macrocephaly who undergo CSF shunting, and who have a high proportion of intracranial CSF compared with brain parenchyma, are still at risk for marked deformity. Once the cranial plate overlap occurs from overdrainage, the bones can become fixed in place. In this circumstance,
\end{abstract}

Abbreviations used in this paper: $\mathrm{P} / \mathrm{L}=$ performance level; $\mathrm{VP}=$ ventriculoperitoneal. previously described delayed reconstruction techniques have carried significant risk, and the results have not always been aesthetically pleasing.

Here we describe our experience with a novel technique to perform a partial cranial reduction and fixation shortly after shunt placement in a newborn with extreme hydrocephalus (Fig. 1). The technique simplified patient positioning; simplified hydrocephalus management, with less frequent valve adjustments; and resulted in an excellent cosmetic result at 1 year.

\section{Case Report}

Birth History and Placement of VP Shunt. This child was born via cesarean section at 39 weeks' gestation, 1 week after the fetus was diagnosed with possible hydran- 


\section{Absorbable skull fixation to prevent shunt-related deformity}

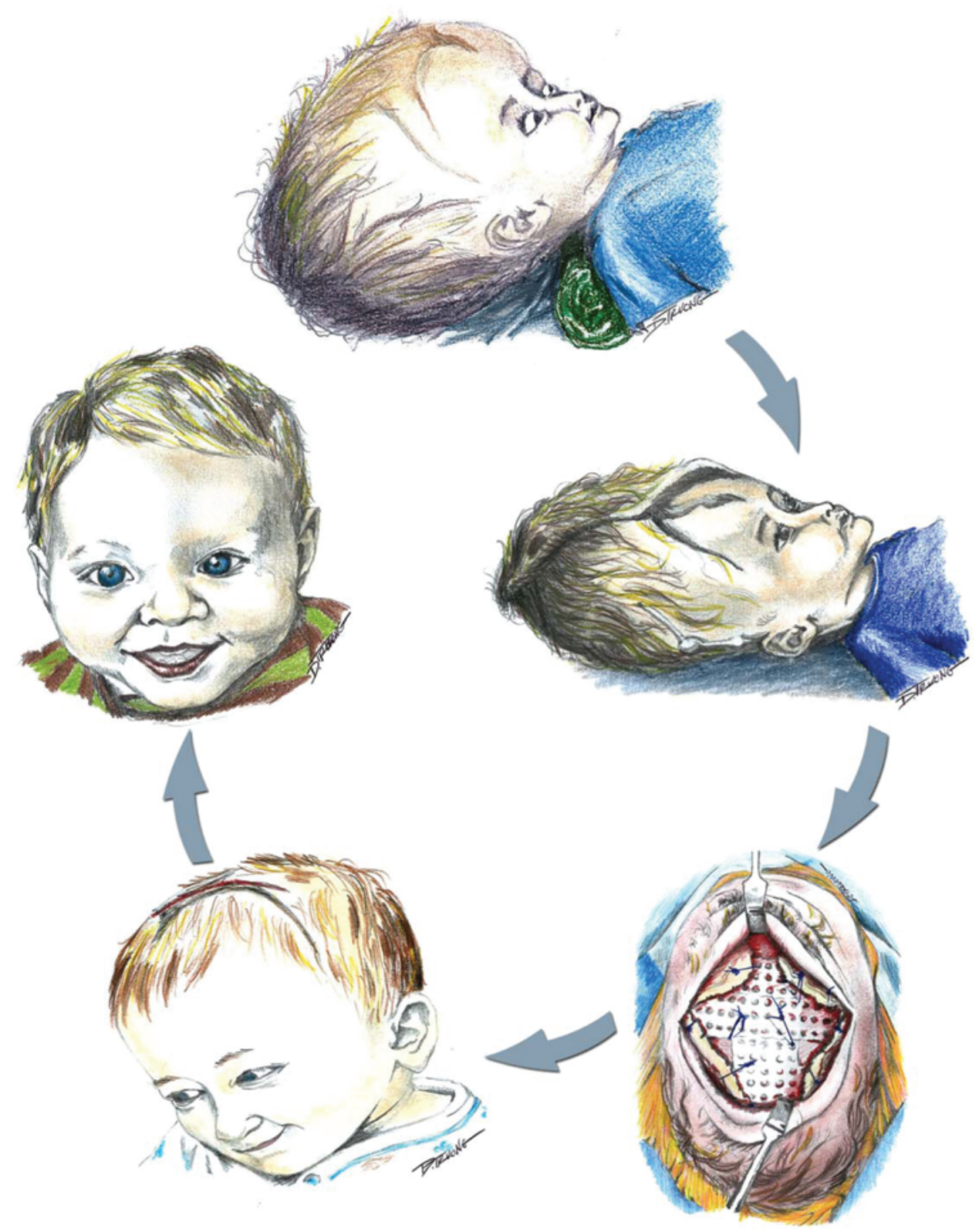

FIG. 1. Artist's graphic summary of the treatment of the child in this case. The uppermost image shows the infant before surgery, with gross macrocephaly and widely split sutures requiring a shoulder roll to support his head and evidence of Parinaud syndrome. The next image, moving clockwise, shows the result after VP shunt placement-a severely collapsed skull with overlapping sutures, dysmorphic appearance, and resolution of Parinaud syndrome. The bottom right image shows an intraoperative vertex view of the reduction and fixation surgery, in which an absorbable plate was placed under the anterior fontanel. The bottom left image shows the result 2 weeks after surgery, with newly formed scar and appearance approaching normalcy. The final image shows the child 14 months after surgery, with an excellent cosmetic outcome.

encephaly by ultrasonography, when the mother noted decreased fetal movement. The Apgar scores were 7 and 9 at 1 and 5 minutes, respectively, and the child did not require mechanical ventilation. The child's head size at birth was $50 \mathrm{~cm}$ (>99th percentile). The child's anterior fontanel was bulging, and the bones of all cranial sutures widely separated, with the parietal bones of the sagittal suture separated by $5 \mathrm{~cm}$.

Initial MRI showed severe hydrocephalus, heterotopic gray matter along the right ventricle, and a very thin cortical mantle (Fig. 2). A right parietal VP shunt, with a
Strata valve (Medtronic) set to P/L 1.5, was placed when the infant was 2 days old. Over the ensuing days, the shunt was reprogrammed to balance CSF drainage. Overdrainage manifested with grossly overlapping sutures and a severely sunken anterior fontanel. Underdrainage manifested with a bulging fontanel and subcutaneous CSF egress around the shunt.

Cranial Reduction Procedure. To avoid a bulging fontanel and/or a pseudomeningocele, the shunt valve was adjusted to a level of increased flow. This resulted 

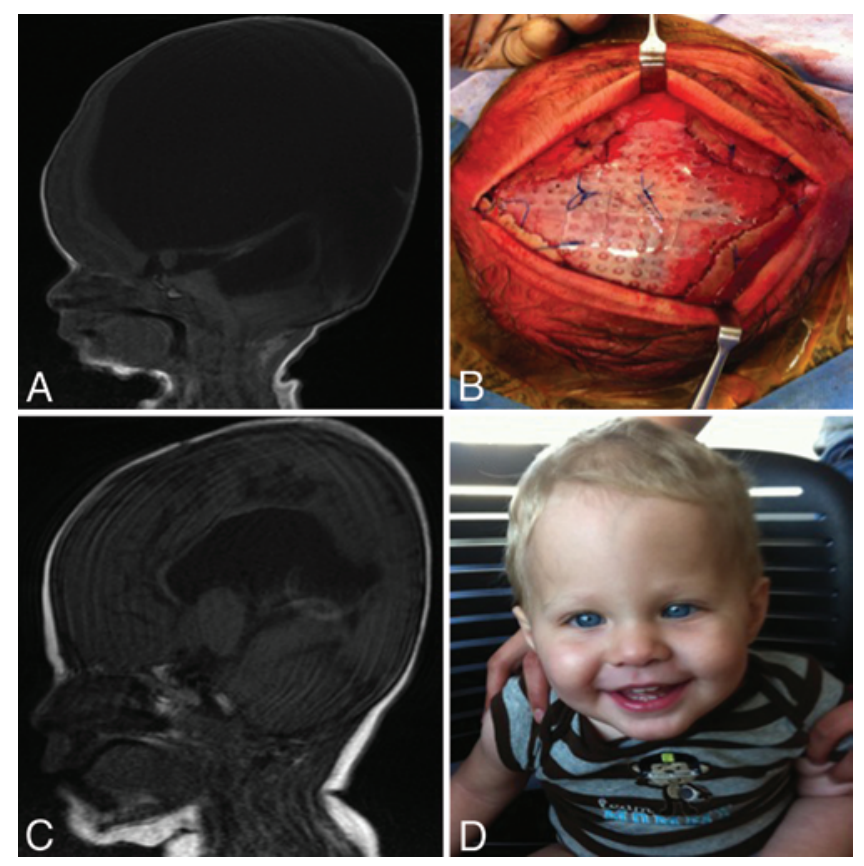

FIG. 2. A: Sagittal preoperative T1-weighted MR image showing severe hydrocephalus and disproportion of face to cranial vault size. B: Intraoperative vertex view of reduction/fixation surgery using an absorbable plate under the anterior fontanel. C: Sagittal postoperative T1-weighted MR image obtained 14 months after surgery depicting normalization of the relative proportions of the face and calvaria and re-expansion of the cortical mantle. D: Frontal photograph obtained 14 months after surgery showing good cosmetic outcome.

in significantly overlapping sutures and the child's head became increasingly dysmorphic, a disturbing appearance for the parents and other health care providers. Positioning the child on his side resulted in a marked bony overlap. For these reasons, the patient was taken back to the operating room in the 1st week of life for a cranial reduction and stabilization procedure.

A limited bicoronal incision was made just behind the large anterior fontanel. This incision allowed access to the frontal bone and both parietal bones as they border the anterior fontanel. The pericranium was scored around the perimeter of the fontanel, allowing establishment of an epidural plane under both parietal and frontal bones. Approximately 20-30 $\mathrm{ml}$ of CSF was aspirated from the lateral ventricle with a 25 -gauge needle through the dura. This low volume reduction allowed for complete apposition of bones along the coronal and sagittal sutures without kinking of the sagittal sinus. The 2 parietal bones were left separated by a few millimeters along the midline, to avoid kinking the superior sagittal sinus. Plicating dural stitches were not placed. The absorbable poly-DL-lactide plate (KLS Martin) was placed in the epidural plane and secured in place to the frontal and parietal bones with absorbable polydioxanone suture (Ethicon) (Fig. 2). We used a single sheet of absorbable plate to rigidly fixate the 4 bones of the anterior fontanel. This step could have been performed with strips of plating material, which would reduce the overall implant burden but would likely have been less secure. Likewise, a 1- to 2-cm hole can be made in the plate as an artificial fontanel.
The child tolerated the 45-minute cranial reduction procedure without complication and with approximately $20 \mathrm{ml}$ of blood loss. This technique was not performed around the posterior fontanel.

The head shape was much improved in the immediate postoperative period and the overall head shape was much less affected by head positioning. Because the occipital bone was not fixated to the parietal bones along the posterior fontanel, we tended to position the patient on the left and right sides, in order to help reduce posterior bone overlap or deformation at the level of the lambdoid suture.

Follow-Up. The child has not required shunt revision and the valve has been maintained at a P/L setting of 1.5. The head circumference was immediately reduced from 50 to $40 \mathrm{~cm}$ after placement of a VP shunt and performance of the cranial reduction and fixation procedure. If we assume that the cranium is roughly spherical, this result represents an approximately $49 \%$ intracranial volume reduction immediately following the reduction/fixation procedure. This volume reduction is in line with reported results for large staged cranial reduction procedures. ${ }^{6,9}$ The head circumference has gradually increased back to $49 \mathrm{~cm}$ over the child's first 17 months of life, with his body size becoming proportional to the head size (Fig. 2). Bone has completely regrown over the anterior fontanel. The ventricles have slowly decreased in size, and the thickness of the cortical mantle has gradually thickened. The patient has had no further cranial surgery, takes no medications, and his development is nearly normal for a child of 17 months. He has slight motor delay, cruising without independent walking. The family is pleased with his appearance and with his development (Fig. 2).

\section{Discussion}

\section{History of Cranial Reduction Surgery for Macrocephaly Related to Hydrocephalus}

Cranial reduction procedures have been reported on a case-by-case or case series basis in the literature for the past 60 years. Multiple forms of craniectomy have been described, most frequently associated with a reduction in cranial volume approximating 50\%-60\%. ${ }^{69}$ Treatment of hydrocephalic severe macrocephaly has historically been undertaken to achieve several goals: 1) to simplify treatment of hydrocephalus, 2) to improve head control, 3) to improve care-taking by easing positioning burdens, 4) to prevent soft-tissue breakdown and infection, and 5) to reduce the magnitude of deformity and improve social acceptance.

The first multistaged cranial reduction procedure for the treatment of severe macrocephaly secondary to hydrocephalus was performed between 1951 and 1953 by Ehni. ${ }^{2}$ The multistage treatment involved 13 procedures within a 22-month period and used novel gap-closing devices invented by the patient's father. This surgery was performed during the initial stages of CSF shunting and did not involve shunt placement.

By the late 1970s, CSF shunting procedures became commonplace and were integrated into the majority of subsequently reported cases of reduction cranioplasty for 
macrocephaly secondary to hydrocephalus. Many authors report placement of VP shunts or subcutaneous ventricle-tapping reservoirs prior to definitive macrocephaly treatment. ${ }^{4,6,7} \mathrm{We}$ advocate the use of a CSF shunt with a programmable valve in this setting. This allows for continued management of the hydrocephalus and management of postoperative subdural hematoma formation in this high-risk group.

Many forms of craniectomy have been described. Vries and $\mathrm{Habal}^{9}$ as well as Ventureyra and Da Silva ${ }^{8}$ removed the entire calvaria and then used a sagittal bandeau to anchor the remaining calvarial pieces to the skull base. Piatt and Arguelles ${ }^{7}$ used a similar technique, but a coronal crossbar was used as the anchor point for reattachment of the calvarial pieces. Park et al. ${ }^{6}$ used the crossbar craniotomy, shaped like a plus sign, to anchor calvarial remnants, and also performed vertical osteotomies. Erdinçler et al. ${ }^{3}$ reported the modified $\Pi$ technique in 1998. Mansour et al. ${ }^{4}$ reported the picket-fence technique and the use of contraction osteogenesis. A thorough written and graphical review of the types of craniectomy and the associated cases was recently contributed by Mathews et al. ${ }^{5}$

Once the craniectomy has been performed, the dura is frequently reduced with an imbricating stitch without kinking the sagittal sinus. It is unclear whether dural plication is necessary to achieve the surgical goals. Postoperative MR venography can be performed to evaluate the status of the sagittal sinus. In our case, the dura was not plicated and dedicated venous imaging was not performed postoperatively.

\section{Early Cranial Fixation in Extreme Hydrocephalus}

To our knowledge, this is the first reported case of very early cranial fixation in extreme hydrocephalus. Since the 1970s, emphasis has been placed on treatment of the hydrocephalus prior to cranial reduction surgery, rather than cranial reduction and fixation surgery functioning as a component of hydrocephalus correction. We devised this operation based on our previous difficulty managing newborn patients with extreme hydrocephalus using shunts alone. Early fixation greatly facilitated patient positioning, immediately improved the infant's appearance, simplified the management of the patient's hydrocephalus, and lessened the potential complications of overdrainage.

Our combined procedure not only facilitated early management of the child, but the reconstruction performed in infancy was far less technically challenging than delayed reconstructions described by numerous previous authors. ${ }^{2-4,7-9}$ Previous reports describe large holocalvarial reconstructions with substantial risk of hemorrhage, transfusion, prolonged time on the operating table, and multiple stages. Our technique limits the entire process of reduction cranioplasty/fixation to 2 steps: placement of a CSF shunt, followed by a single cranial remodeling and fixation procedure that takes less than 1 hour. It is even possible that future modifications of this procedure may allow for a smaller incision, the use of smaller plates, and the possibility of combining the cranial fixation and shunt placement into a single procedure.

\section{Conclusions}

Early shunting combined with a limited cranial reduction and fixation procedure facilitated patient positioning, simplified management of hydrocephalus, and provided an excellent aesthetic outcome in an infant with extreme macrocephaly related to hydrocephalus.

\section{Disclosure}

The authors report no conflict of interest concerning the materials and methods used in this study or the findings specified in this paper.

Author contributions to the study and manuscript preparation include the following. Conception and design: Tuite, Truong. Acquisition of data: Tuite, Manwaring, Deukmedjian, Carey, Tetreault. Analysis and interpretation of data: Manwaring. Drafting the article: Tuite, Manwaring. Critically revising the article: all authors. Reviewed submitted version of manuscript: all authors. Approved the final version of the manuscript on behalf of all authors: Tuite. Performed surgery: Tuite, Deukmedjian, Carey. Illustrations: Truong.

\section{Acknowledgments}

The authors thank Christine Hancock for her administrative assistance and Brian Rockwood for his supply of the resorbable plate.

\section{References}

1. Doorenbosch X, Molloy CJ, David DJ, Santoreneos S, Anderson PJ: Management of cranial deformity following ventricular shunting. Childs Nerv Syst 25:871-874, 2009

2. Ehni G: Reduction of head size in advanced hydrocephalus: a case report. Neurosurgery 11:223-228, 1982

3. Erdinçler P, Kaynar MY, Canbaz B, Etus V, Ciplak N, Kuday C: Two different surgical techniques for reduction cranioplasty. Childs Nerv Syst 14:372-377, 1998

4. Mansour N, Sobel L, Lee M, Larumbe J, Stelnicki E: A new method for the treatment of macrocephaly caused by hydrocephalus. Cleft Palate Craniofac J 42:1-6, 2005

5. Mathews MS, Loudon WG, Muhonen MG, Sundine MJ: Vault reduction cranioplasty for extreme hydrocephalic macrocephaly. J Neurosurg 107 (4 Suppl):332-337, 2007

6. Park TS, Grady MS, Persing JA, Delashaw JB: One-stage reduction cranioplasty for macrocephaly associated with advanced hydrocephalus. Neurosurgery 17:506-509, 1985

7. Piatt JH Jr, Arguelles JH: Reduction cranioplasty for craniocerebral disproportion in infancy: indications and technique. Pediatr Neurosurg 16:265-270, 1990-1991

8. Ventureyra EC, Da Silva VF: Reduction cranioplasty for neglected hydrocephalus. Surg Neurol 15:236-238, 1981

9. Vries JK, Habal MB: Cranio-orbital correction for massive enlargement of the cranial vault. Plast Reconstr Surg 63:466-472, 1979

Manuscript submitted June 28, 2012.

Accepted October 31, 2012.

Please include this information when citing this paper: published online December 11, 2012; DOI: 10.3171/2012.10.PEDS12340.

Address correspondence to: Gerald Tuite, M.D., All Children's Hospital Neuroscience Institute, 601 5th Street South, Suite 511, St. Petersburg, Florida 33701. email: geraldtuite@ gmail.com. 